\title{
Vigabatrin and lamotrigine in refractory epilepsy
}

\author{
Iwona Stolarek, Jacqueline Blacklaw, Gerard Forrest, Martin J Brodie
}

Epilepsy Research Unit, University Department of Medicine and Therapeutics, Western Infirmary, Glasgow G11 6NT, UK

I Stolarek

J Blacklaw

G Forrest

M J Brodie

Correspondence to: Dr M J Brodie.

Received 2 August 1993 and in revised form 25 October 1993.

Accepted 12 November 1993

\begin{abstract}
Epilepsy arises from an imbalance of inhibitory and excitatory influences in the brain. Vigabatrin (VIG) decreases the breakdown of the inhibitory neurotransmitter $\gamma$-aminobutyric acid, whereas lamotrigine (LTG) reduces presynaptic excitatory amino acid release. 22 patients with refractory epilepsy, treated with an anticonvulsant regimen containing VIG, entered a balanced, double blind, placebo controlled, crossover trial of additional LTG. Treatment periods of 12 weeks (25 mg, $50 \mathrm{mg}, 100 \mathrm{mg}$ LTG twice daily for four weeks at each dose, and matched placebo) were followed by wash out intervals of four weeks. 14 of the 20 patients completing the study improved, resulting in a significant fall in seizure days and numbers. Analysis of seizure type confirmed a beneficial effect on partial and secondary generalised tonic-clonic seizures. At the highest LTG dose $(200$ mg daily) there was a median fall of $37 \%$ in seizure count with nine $(45 \%)$ patients reporting $>50 \%$ reduction. Three of these patients were seizure free during this month of treatment. Side effects were minimal throughout the study. Concentrations of other antiepileptic drugs, including those of carbamazepine 10,11-epoxide, were not modified by LTG. This study suggests a substantial efficacy for a regimen containing VIG and LTG. Combinations of drugs with complementary modes of action may provide a rational pharmacological approach to the management of refractory epilepsy.
\end{abstract}

(F Neurol Neurosurg Psychiatry 1994;57:921-924)

decreasing presynaptic excitatory amino acid release. ${ }^{2}$ Anecdotal reports about three patients suggested substantial efficacy for VIG and LTG in combination, when individual treatment with either drug had failed. ${ }^{34}$ Our objective was to explore this finding further with a placebo controlled, dose ranging study of additional LTG in patients with refractory epilepsy and receiving an anticonvulsant regimen containing VIG. As neither drug has a product licence for use as monotherapy, it was not possible to undertake the study in patients receiving vigabatrin alone.

\section{Patients and methods}

PATIENTS

Twenty two patients with complex partial seizures with or without secondary generalisation were recruited. All had reported a minimum of three seizures a month for the previous three months despite a stable regimen of anticonvulsant treatment. Nine and 13 patients took VIG with one or two other antiepileptic drugs respectively. All gave written informed consent to their participation in the trial, which had local ethics committee approval.

PROTOCOL

This was a randomised, double blind, placebo controlled, crossover, dose ranging study of additional LTG. After an initial four week run in, two 12 week treatment periods $(25 \mathrm{mg}, 50$ $\mathrm{mg}$, and $100 \mathrm{mg} \mathrm{LTG}$ twice daily for four weeks each and matched placebo) were followed by four-week washout intervals. Patients were examined at weeks $0,4,8$, and 12 during both treatments. Appointment times were individualised and kept constant. Medication was taken at the same times each day. Compliance was checked by a tablet count.

Seizures follow abnormal synchronisation and amplification of neuronal firing in electrically unstable areas of the brain. They may arise from an imbalance of inhibitory and excitatory influences at a cellular level, resulting in either generation of electrical activity or in prevention of its inhibition. ${ }^{1}$ The major inhibitory neurotransmitter in the human brain is $\gamma$-aminobutyric acid (GABA) and the principal fast excitatory neurotransmitter is glutamate.

Two new antiepileptic drugs, vigabatrin (VIG) and lamotrigine (LTG), redress this presumed chemical imbalance, the first by increasing brain GABA and the second by 
Median seizure numbers in 20 patients with refractory epilepsy after treatment with LTG and matched placebo. Horizontal bars are $95 \%$ CIs of the differences.

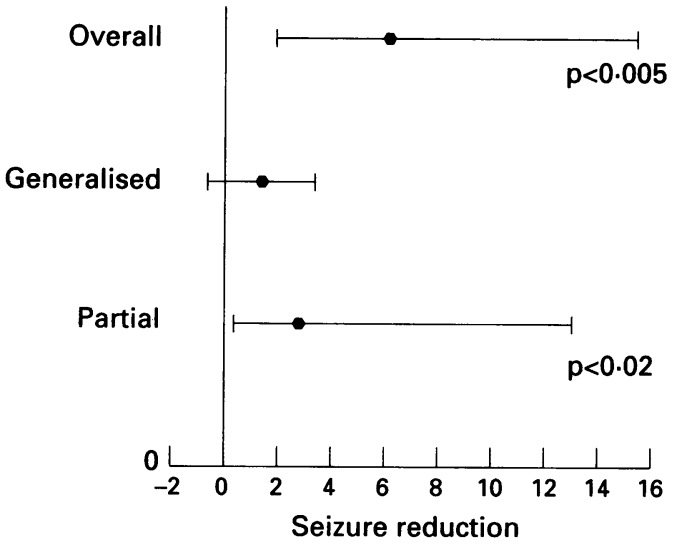

(unsteadiness, headache, dizziness, double vision, nausea, tremor) were commonly associated with anticonvulsant treatment. Also, 10 $\mathrm{cm}$ visual analogue scales measuring subjective sedation, concentration, memory, and mood were completed by the patients at each visit. At the end of the study each patient was invited to state a preference for one or other treatment period before the code was broken.

\section{DRUG ASSAYS}

Vigabatrin was extracted from plasma into ethylacetate, heated with dansyl chloride at high $\mathrm{pH}$ to form a fluorescent derivative, and measured by high performance liquid chromatography (HPLC) with phenylGABA as internal standard. The interassay coefficient of variation (CV) over the range $1-100 \mathrm{mg} / 1$ was $5 \%$ and the lower limit of detection was $0.1 \mathrm{mg} / 1 .^{5}$ Lamotrigine was extracted into ethinyl acetate from plasma with $2 \mathrm{M}$ sodium hydroxide and measured by HPLC with BWA 725C (Wellcome Laboratories, UK) as internal standard. The interassay $\mathrm{CV}$ over the range $0-5 \mathrm{mg} / 1$ was $6 \%$ with a lower limit of detection of $0.25 \mathrm{mg} / \mathrm{l}$. Carbamazepine, sodium valproate, phenytoin, and phenobar-

Table 1 Median seizure numbers (range) and reductions (95\% CIs of the difference) in 20 patients with refractory epilepsy receiving adjuvant $L T G$ and matched placebo

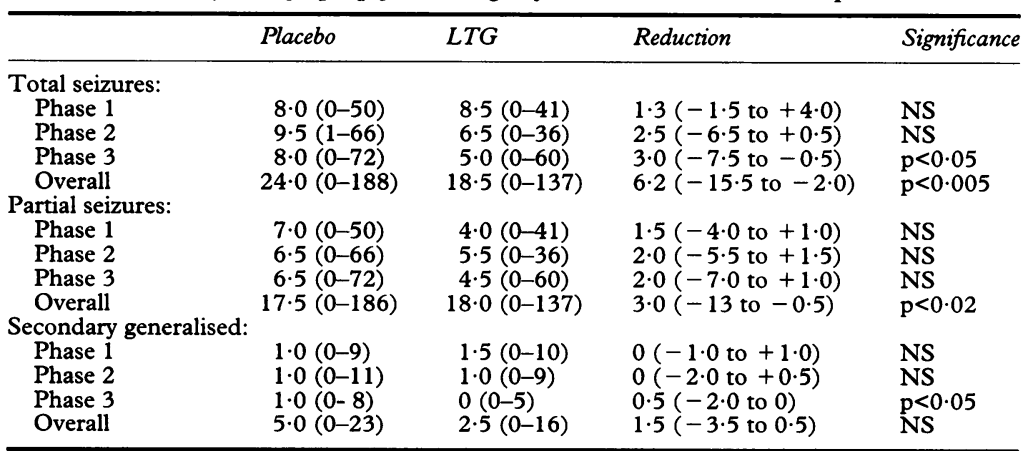

Phase 1, 25 mg LTG twice daily; phase 2, 50 mg LTG twice daily; phase 3, 100 mg LTG twice daily.

Table 2 Reduction in mean seizure counts and numbers of responders during adjunct $L T G v$ placebo in 20 patients with refractory epilepsy

\begin{tabular}{llll}
\hline & \multicolumn{3}{l}{ No of responders } \\
\cline { 3 - 4 } \cline { 3 - 3 } & $\begin{array}{l}\text { Seizure } \\
\text { reduction }\end{array}$ & $\begin{array}{l}>25 \% \\
\text { reduction }\end{array}$ & $>50 \%$ \\
& $-6 \%$ & $5(25 \%)$ & $3(15 \%)$ \\
& $32 \%$ & $4(20 \%)$ & $7(35 \%)$ \\
Phase 1 (25 mg twice daily) & $37 \%$ & $3(15 \%)$ & $9(45 \%)$ \\
Phase 2 (50 mg twice daily) & $23 \%$ & $6(30 \%)$ & $4(20 \%)$ \\
Phase 3 (100 mg twice daily) & Overall & &
\end{tabular}

bitone (in patients taking primidone) concentrations were measured by enzyme immunoassay (Emit, Syva, Palo Alto, USA). The active metabolite, carbamazepine 10, 11 epoxide, was determined by HPLC with 5-(p methylphenyl 1)-5-phenyl hydantoin as internal standard. ${ }^{6}$

STATISTICS

Statistical analysis of seizure data, adverse effects, and antiepileptic drug concentrations was performed with the Wilcoxon rank test for matched pairs. The $95 \%$ confidence intervals (95\% CIs) for the differences were calculated around median values with the Minitab statistical package. Mann-Whitney analysis for an order effect was carried out for total, partial, and generalised seizures.

\section{Results}

\section{PATIENTS}

Twenty patients (eight men, 12 women) completed the trial. One withdrew due to transport difficulties in attending the hospital and another because of side effects (tiredness, dizziness, sore throat, emotional lability, irritability, weepiness, depression) while taking the placebo. Equal numbers of patients were randomised first to both arms of the study and there was no evidence of an order effect for any of the data.

\section{SEIZURES}

There was a significant reduction in seizure days during LTG treatment compared with placebo (median 5.5, p $<0.01,95 \% \mathrm{CI}-10$ to -1.5$)$, particularly at the highest dose (median 2.5 days, $\mathrm{p}<0.05,95 \% \mathrm{CI}-5.0$ to $0)$. Seizure numbers were reduced overall by $23 \%$ (figure, $\mathrm{p}<0.005,95 \% \mathrm{CI}-15.5$ to $-2)$. Subtype analysis showed a fall in partial ( $\mathrm{n}=20$, median $3, \mathrm{p}<0.02,95 \% \mathrm{CI}-13$ to $-0.5)$ and secondary generalised tonic-clonic seizures ( $n=15$, median 1.5 , NS, 95\% CI -3.5 to 0.5 ) (table 1 ). At the highest LTG dose (200 mg daily) there was an overall reduction of $37 \%$ in seizure numbers with nine $(45 \%)$ patients reporting a fall of more than $50 \%$ compared with the equivalent placebo phase (table 2). Three patients remained seizure free during this month of treatment.

As the LTG dosage was titrated, results in the phases (39/60) during which LTG plasma concentrations exceeded $1 \mathrm{mg} / 1$ were compared with the equivalent periods on placebo. This analysis excluded data from 15 patients during phase 1 (LTG $25 \mathrm{mg}$ twice daily) and seven patients during phase $2(50 \mathrm{mg}$ twice daily). Overall, there was a fall of $30 \%$ in seizure count. Sixteen patients improved with seven $(35 \%)$ reporting $>50 \%$ reduction. A significant fall was found for total (median $4.5, \mathrm{p}<0.01,95 \% \mathrm{CI}-8.5$ to -2.0$)$ and secondary generalised (median $2, \mathrm{p}<0.02$, $95 \% \mathrm{CI}-3.5$ to 0 ) seizure numbers. The reduction in partial seizures failed to reach significance (median $2, \mathrm{NS}, 95 \% \mathrm{CI}-6.5$ to $+0 \cdot 5)$. 
Table 3 Mean (SD) anticonvulsant concentrations ( $m g / l)$ in 20 patients receiving four weeks' adjuvant treatment with $100 \mathrm{mg}$ LTG twice daily and matched placebo

\begin{tabular}{lccc}
\hline Anticonvulsant & No & Placebo & LTG \\
\hline Vigabatrin & 20 & $26(21)$ & $26(21)$ \\
Carbamazepine & 15 & $8 \cdot 7(2 \cdot 8)$ & $7 \cdot 6(2 \cdot 1)$ \\
Carbamazepine 10, 11 epoxide & 15 & $1 \cdot 2(0 \cdot 6)$ & $1 \cdot 2(0 \cdot 8)$ \\
Sodium valproate & 5 & $68(18)$ & $69(30)$ \\
Phenytoin & 3 & $14(2 \cdot 4)$ & $14(1 \cdot 1)$ \\
\hline
\end{tabular}

SIDE EFFECTS

There were no significant differences in the numbers of spontaneous or requested side effects reported during the two treatment periods (spontaneous: LTG six, placebo seven; requested: LTG six, placebo seven). No one side effect was reported more often with LTG than placebo. Mean visual analogue scores for sedation, concentration, memory, and depression did not differ significantly after a month's treatment between LTG (100 mg twice daily) and matched placebo (data not shown). Sixteen patients preferred LTG treatment, whereas three of the remaining four chose placebo $(p<0.001)$.

\section{CONCENTRATIONS}

Concentrations of LTG (median (range)) after each treatment phase varied substantially among the patients (phase 1: $0.64 \mathrm{mg} / 1$ $(0 \cdot 34-2 \cdot 7)$; phase $2: 1.2 \mathrm{mg} / 1 \quad(0 \cdot 47-4 \cdot 1)$; phase 3: $2.4 \mathrm{mg} / 1(1-7 \cdot 9))$. The mean LTG concentration in the nine patients reporting $>50 \%$ seizure reduction during phase 3 was higher than in the 11 non-responders (3.2 $\mathrm{mg} / \mathrm{l} v 2 \cdot 1 \mathrm{mg} / \mathrm{l})$, but not significantly so. No significant differences were found after the highest LTG dose for concentrations of concomitant anticonvulsants or of the active metabolite carbamazepine 10,11 epoxide (table 3).

\section{Discussion}

Vigabatrin binds irreversibly to the enzyme GABA transaminase preventing GABA breakdown and increasing brain concentrations of this important inhibitory neurotransmitter. ${ }^{7}$ In placebo controlled trials, treatment with VIG produced a sustained reduction in seizure numbers in around $50 \%$ of patients with refractory epilepsy. Clinical studies with this drug, however, suggested better efficacy against partial than generalised tonic-clonic seizures.

Lamotrigine is a phenyltriazine compound that reduces presynaptic excitatory amino acid release by blocking voltage sensitive sodium channels, thus attenuating neuronal excitation. ${ }^{8}$ Eight double blind, placebo controlled, add on, crossover trials have investigated the efficacy of LTG in refractory epilepsy. ${ }^{9-16}$ Seven of these showed a significant reduction in total and partial seizure counts. ${ }^{9}{ }^{1012-16}$ In three, there were sufficient numbers of patients with secondary generalised tonic-clonic seizures to confirm a beneficial effect on this seizure type also. ${ }^{91114}$

Side effects during our present study were minimal with no statistical difference noted between LTG and placebo. Mean linear analogue scores for sedation, concentration, memory, and depression were no different after a month's treatment with highest LTG dose and placebo. Mood lifting effects with LTG have been reported anecdotally in several studies. ${ }^{8}$ In another, it has been suggested that quality of life is enhanced by LTG treatment, although this may simply be a function of reduced seizure numbers and severity. ${ }^{14}$

Other antiepileptic drug concentrations were unaffected by LTG. In particular, there was no change in carbamazepine 10, 11 epoxide, the active metabolite of carbamazepine, which was thought to increase in a recent open study with similar LTG doses. ${ }^{17} \mathrm{We}$ therefore postulate a pharmacodynamic mechanism for the well described interaction between LTG and carbamazepine.

This small study suggests substantial efficacy for an antiepileptic drug regimen containing VGB and LTG with a significant overall reduction in seizure days and numbers. Analysis of seizure type confirmed an effect on both partial and secondary generalised seizures. On the highest LTG dose (200 mg daily), $45 \%$ of patients documented at least $50 \%$ seizure reduction. The mean equivalent figure for the eight published placebo controlled, crossover trials is $22 \% .^{18}$ In a parallel group study among 216 patients with refractory partial seizures randomly assigned to an additional $300 \mathrm{mg}$ or $500 \mathrm{mg}$ LTG daily or placebo, only the higher LTG dose resulted in a significant fall in seizure numbers. ${ }^{19}$ The $500 \mathrm{mg}$ dose produced a $50 \%$ seizure reduction in only $34 \%$ of patients compared with $45 \%$ with $200 \mathrm{mg}$ LTG daily in our study.

The maximum dose of LTG employed in our study was only $200 \mathrm{mg}$ daily, which is lower than that used in most published trials. Nevertheless, this resulted in a fall in seizures of $37 \%$ with $45 \%$ of the patients reporting greater than $50 \%$ seizure reduction. Scope, therefore, remained to increase the LTG dose further. Eight of the responders are currently taking 400-500 mg LTG daily and undergoing withdrawal of their standard anticonvulsant drugs to leave them on VIG and LTG only. So far, five of these patients are seizure free. Interestingly, synergism has been suggested for LTG in combination with sodium valproate, another drug thought to act by potentiating GABA-ergic inhibition. ${ }^{20}{ }^{21}$ Combining drugs with complementary modes of action may provide a rational pharmacological approach to the management of refractory epilepsy.

Our thanks go to Mrs Moya Dewar for expert secretarial assistance and to Dr AWC Yuen from the Wellcome Trust for supplying the lamotrigine and placebo tablets.

1 Meldrum BS. Anatomy, physiology and pathology of epilepsy. Lancet 1990;336:231-4.

2 Leach JP, Brodie MI. Novel antiepileptic drugs. In Clifford-Rose F, ed. New advances in neuropharmacology. Clifford-Rose F, ed. New advances in neurc

3 Kirker A, Reynolds EH. Vigabatrin and lamotrigine in a patient with an intractable epilepsy. Acta Neurol Scand 1990;82 Suppl 133:S38-9. 
4 Stewart J, Hughes E, Reynolds EH. Lamotrigine for generalised epilepsies. Lancet 1992;340:1223.

5 McKee PJW, Blacklaw J, Friel E, Thompson GG, Gillham RA, Brodie MJ. Adjuvant vigabatrin in refractory epilepsy: a ceiling to effective dosage in individual patients? Epilepsia 1993;34:937-43.

6 Macphee GJA, Thompson GG, Scobie G, et al. Effects of cimetidine on carbamazepine auto- and heteroinduction in man. Br f Clin Pharmacol 1984;18:411-9.

7 Grant SM, Heel RC. Vigabatrin. Drugs 1992;41:889-926.

8 Brodie MJ. Lamotrigine. Lancet 1992;339:1397-400.

9 Jawad S, Richens A, Goodwin G, Yuen AWC. Controlled trial of lamotrigine (Lamictal) for refractory epileptic trial of lamotrigine (Lamictal) fo

10 Binnie CD, Debets RMC, Engelsman M, et al. Doubleblind crossover trial of lamotrigine (Lamictal) as add-on therapy in intractable epilepsy. Epilepsy Res 1989;4 222-9.

11 Sander JWAS, Patsalos PN, Oxley JR, Hamilton MJ, Yuen AWC. A randomised double-blind, placebo-controlled, add-on trial of lamotrigine in patients with severe epilepsy. Epilepsy Res 1990;6:221-6.

12 Loiseau P, Yuen AWC, Duche B, Menager T, Arné-Bès MC. A randomised double-blind, placebo-controlled, crossover, add-on trial of lamotrigine in patients with treatment resistant partial seizures. Epilepsy Res 1990;7: 136-45.

13 Messenheimer $\mathrm{J}$ and the Lamictal Study Group. Effectiveness of lamotrigine (Lamictal) in patients with refractory partial seizures [abstract]. Epilepsia 1991;32 Suppl 1:57

14 Smith D, Baker G, Davies G, et al. A placebo-controlled, double-blind, crossover trial of lamotrigine as add-on therapy on seizure frequency, severity, mood and quality of life in patients with treatment-resistant epilepsy. $f$ Neurol Neurosurg Psychiatry 1992;55:416-23.

15 Schapal GJ, Beran RG, Vaida FJE, et al. Double-blind, placebo-controlled, crossover study of lamotrigine in treatment-resistant partial seizures. 7 Neurol Neurosurg Psychiatry 1993;56:448-53.

16 Schmidt D, Ried S, Rapp P. Add-on treatment with lamotrigine for intractable partial epilepsy: a placeborigine for intractable partial epilepsy: a placebo-
controlled cross-over trial. Epilepsia 1993;34:Suppl 2:66.

17 Warner T, Patsalos PN, Elyas AA, Duncan JS. Lamotrigine-induced carbamazepine toxicity: an interaction with carbamazepine 10, 11-epoxide. Epilepsy Res 1992;11:147-50

18 Goa KL, Ross SR, Chrisp P. Lamotrigine: a review of its pharmacological properties and clinical efficacy in epilepsy. Drugs 1993;46:152-76.

19 Matsuo F, Bergen D, Faught E, et al. Placebo-controlled study of the efficacy and safety of lamotrigine in patients with partial seizures. Neurology 1993;43:2284-91.

20 Panayiotopoulos CP, Ferrie CD, Knott C, Robinson RO. Interaction of lamotrigine with sodium valproate. Lancet 1993;341:445.

21 Pisani F, Di Perri R, Perucca E, Richens A. Interaction of lamotrigine with sodium valproate. Lancet 1993;341:1224. 